\title{
Verlaufsbeobachtung nach Mittelfußköpfchenresektion nach Lelièvre
}

\author{
J. Grifka ${ }^{1}$, O. Oest $t^{2}$ \\ 'Orthopädische Universitätsklinik im St. Josef-Hospital, 4630 Bochum (Direktor: Prof. Dr. med. J. Krämer) \\ ${ }^{2}$ Orthopädische Klinik des evang. Fachkrankenhauses, 4030 Ratingen
}

Gewidmet: Herrn Prof. Dr. K. H. Idelberger zum 80. Geburtstag

\section{Zusammenfassung}

Bei ausgeprägten Vorfußdeformitäten wurde in der orthopädischen Klinik des evangelischen Fachkrankenhauses Ratingen von 1975 bis 1987 in 107 Fällen die Mittelfußköpfchenresektion nach Lelièvre durchgeführt. Für den Behandlungserfolg entscheidende Besonderheiten des operativen Vorgehens und insbesondere die Erfordernisse der postoperativen Nachbehandlung werden verdeutlicht. Mögliche Komplikationen des Heilungserfolges werden dargestellt. Die Mittelfußköpfchenresektion nach Lelièvre erweist sich als geeignete Maßnahme zur Verhinderung der Immobilität, vor allem bei fortgeschrittenen, entzündlichen Gelenkveränderungen im Vorfußbereich.

\section{Follow-up After Removal of the Head of the Metacarpal Bone After Lelièvre}

From 1975 to 1987 on 107 cases of severe deformities of the forefoot a resection of the metatarsal heads according to the method of Lelièvre was performed. As to the success of this method special features of this operation and especially the requirements of postoperative treatment will be explained. Possible complications of healing will be detailed. The procedure of resecting the heads of the metatarsal bones according to Lelièvre seems to be recommendable in order to prevent immovability, especially in case of advanced inflammable alterations of the joints in the forefoot.

\section{Einleitung}

Ausgeprägte Vorfußdeformitäten sind bei rheumatischen Erkrankungen häufig. Nach Tillmann (9), zeigt sich der Befall der Metatarsophalangealgelenke oft als Ort der Erstmanifestation.

Wegen der ausgeprägten Schmerzsymptomatik mit Bewegungs- und Belastungsschmerz, können diese Veränderungen bis zur völligen Gehunfähigkeit führen. Dies kann sogar bei Patienten, die sich ohne ihr Fußleiden selbst versorgen könnten, bis zur Bettlägrigkeit und Pflegebedürftigkeit führen.

Z. Orthop. 127 (1989) 561-565

(C) 1989 F. Enke Verlag Stuttgart

\section{Material und Methode}

Mit dem Ziel, bei den massiven Gelenkveränderungen Schmerzfreiheit und gute Belastbarkeit herbeizuführen, wurde von 1975 bis 1987 bei 107 Füßen die Mittelfußköpfchenresektion nach Lelièvre (6) durchgeführt. Das durchschnittliche Lebensalter bei Operation betrug 60 (29-79) Jahre. Bei sämtlichen Fällen wurde eine Auswertung der Krankenunterlagen vorgenommen. Bei 64 Füßen konnte nach durchschnittlich 5,3 (1-11) Jahren eine Nachuntersuchung erfolgen.

Entsprechend der Angabe von Lelièvre (6) wurden die Mittelfußköpfchen subkapital reseziert und die phalangeale Grundgliedbasis belassen. Der operative $\mathrm{Zu}$ gang wurde unterschiedlich gewählt. In 48 Fällen wurde der dorsale Zugang verwandt, in 59 Fällen der plantare mit einem bogenförmigen Schnitt ohne Hautexzision und Dermodese. Der Zugang zum ersten Strahl wurde entsprechend der Keller-Brandes-Operation mediodorsal gewählt. Der Zugang zur Großzehe ist nach erfolgtem Eingriff an den Metatarsalia II-V erleichtert, da die Weichteile entspannt sind und der erste Strahl somit gut dargestellt werden kann. Nur in Einzelfällen war es notwendig, wegen der erheblichen Lateraldeviation der Großzehe, die nahezu quer zu den anderen Zehen stand und diese verdrängte, zunächst den Eingriff am I. Strahl vorzunehmen, bevor die übrigen Köpfchen reseziert werden konnten. Am I. Strahl wurde die Grundgliedbasis reseziert und das erste Mittelfußköpfchen modelliert mit Abtragung der medialseitigen Pseudoexostose. Dabei wurde angestrebt, daß am Mittelfußköpfchen lediglich soviel abgetragen wurde, daß die beiden Sesambeine noch ein Gegenlager gegen das Köpfchen hatten, wie es Lelièvre (6) fordert, um die für die Abrollung wichtige Belastbarkeit auf dem I. Strahl zu gewährleisten.

Postoperativ wurde die Zehenstellung passager mit Kirschner-Drähten oder im Tennisschläger-Gips (Abb. 1) fixiert. Die Mobilisierung erfolgte unter Fersenbelastung mit der Berkemann-Fersensandale, die eine reine Fersenbelastung bei Entlastung des Vorfußes ermöglicht (Abb. 2). Nach der initialen postoperativen Zehenfixation über zwei Wochen wurden die Patienten dazu angeleitet, einen zehenschienenden Verband anzulegen. Ein Teil der Patienten wurde mit individuell gefertigten Schienen (Abb. 3 ) in einer Modifikation der von Gschwend (5) verwendeten Fußschienen versorgt. Dazu wurde ein Orthoplastmaterial am Fuß geformt und innenseitig im Vorfuß- und Zehenbereich gepolstert. Die Zehen werden durch einen nochmals gepolsterten Klettverschluß druckfrei gezügelt. 


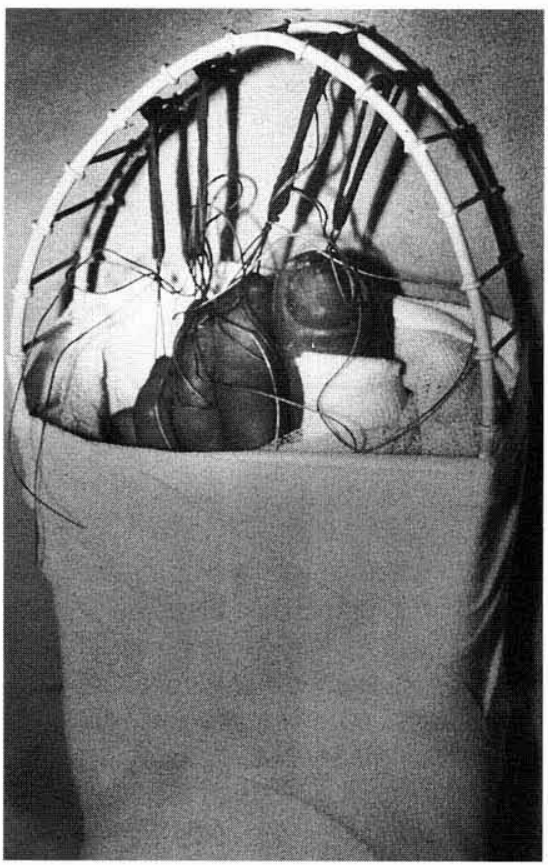

Abb. 1 Initial postoperative Fixation der Zehenstellung und Distraktion der Resektionsbezirke

a) im Tennisschlägergips

b) mit parossären Kirschner-Drähten

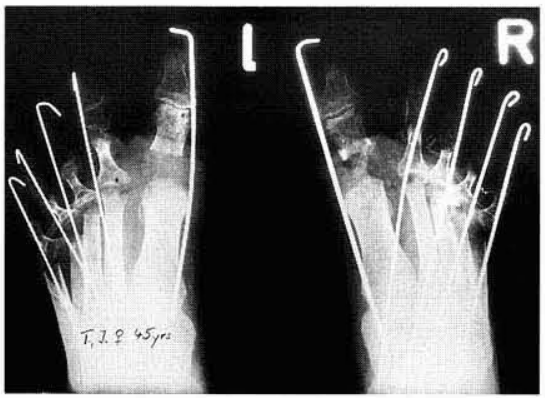

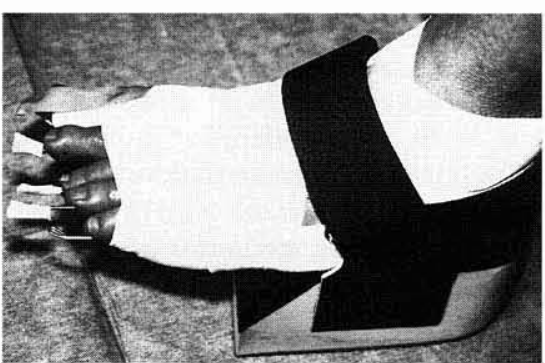

Abb. 2

Berkemann-Fersensandale zur postoperativen Mobilisierung mit Fersenbelastung bei Entlastung des Vorfußes

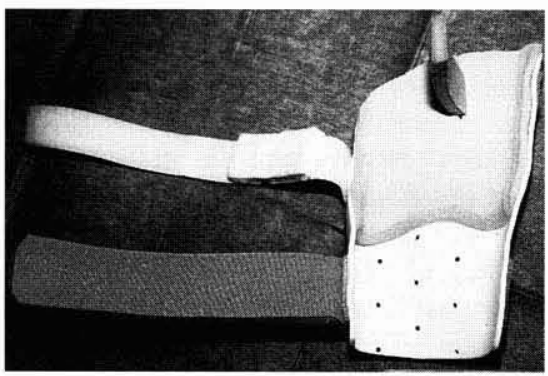

Abb. 3

Individuell gefertigte Vorfußschiene zur Haltung der Zehenstellung

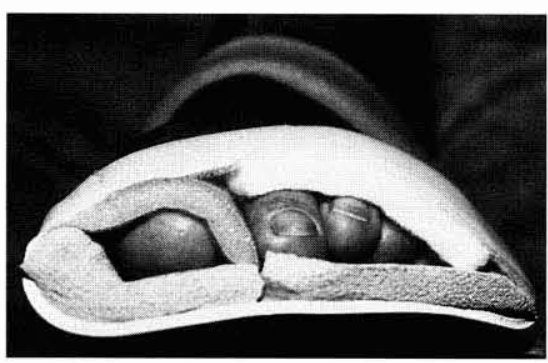

\section{Ergebnisse}

Das durch die Operation erzielte Gesamtergebnis wurde von $72 \%$ der Patienten als gut oder sehr gut bewertet. $85 \%$ gaben an, daß sie die Behandlung, unter Berücksichtigung der vorherigen Beschwerden und Einschränkungen erneut wieder vornehmen lassen würden. Als Grund für eine Ablehnung der nochmaligen Behandlung wurden die lange Zeitdauer und Komplikationen im Behandlungsverlauf genannt. Die Rate der Sekundärheilungen war mit $15 \%$ beim dorsalen Zugang und $16 \%$ beim plantaren Zugang gegenüber der üblichen Rate von Wundheilungsstörungen deutlich erhöht mit gleichmäßiger Verteilung auf beide Zugänge (Tab. 1). Ein Revisionseingriff wegen Infizierung war in keinem Fall erforderlich.

Beim dorsalen Zugang war in fast $40 \%$ die Zehenbeweglichkeit aufgehoben; zumeist aufgrund von Narbenkontrakturen. In diesen Fällen waren die Zehen mit Abstand von der Bodenfläche fixiert.

Tab. 1 Vergleich zwischen dorsalem und plantarem Zugang

\begin{tabular}{l|l|l} 
Vergleich des operativen Zuganges & dorsal & plantar \\
\hline Heilung per secundam & $15 \%$ & $16 \%$ \\
$\begin{array}{l}\text { Zehenbeweglichkeit } \\
\quad \text { gut oder }\end{array}$ & & \\
$\quad$ Zufriedenstellend & $63 \%$ & $74 \%$ \\
$\quad$ nicht ausreichend & $37 \%$ & $26 \%$ \\
$\begin{array}{l}\text { kosmetisch unbefriedigend } \\
\quad \text { (Patientenurteil) }\end{array}$ & $7 \%$ & $15 \%$ \\
Narbe gereizt & $0 \%$ & $6 \%$
\end{tabular}


Die erreichte kosmetische Korrektur der Fußform und Zehenstellung wurde von den Patienten selbst meist positiv beurteilt. Beim plantaren Zugang war die Rate der unzufriedenen Patienten mit $15 \%$ doppelt so hoch wie beim dorsalen, ohne daß vom kosmetischen Resultat ein objektiver Unterschied feststellbar war. In der Nachkontrolle fand sich bei $6 \%$ der von plantar Operierten eine Narbenreizung, während das beim dorsalen Zugang in keinem Fall vorlag. Diese Patienten stuften auch das kosmetische Ergebnis als schlecht ein. In diesen Fällen einer verbleibenden Narbenreizung und Belastungsschmerzen im Narbenbereich war die Narbe in der Belastungsfläche unter den Metatarsalestümpfen lokalisiert und war somit dem Belastungsdruck ausgesetzt.

In der vorliegenden Untersuchung ließ sich keine Korrelation zwischen der Zufriedenheit der Patienten und dem Verhältnis des Metatarsale-Index von praeoperativ zu postoperativ feststellen. Es zeigte sich aber, daß Patienten mit einem postoperativen Alignement mit längerbelassenem ersten Metarsale in höherem Maße subjektive Zufriedenheit angaben.

Bei 14 Patienten, die einen Druckschmerz unter den mittleren Metatarsalia angaben, war entweder kein regelrechtes Alignement geschaffen worden oder einzelne Metatarsalestümpfe wiesen bei scheinbarem Alignement in der a. p. Röntgenprojektion ein fixiertes Tiefertreten nach plantar in die Belastungsfläche auf. Die vermehrte Druckbelastung unter den betroffenen, aus der Reihe zu weit nach distal und plantar vorstehenden Mittelfußstümpfen wurde mit Trittspurbildern (Abb. 4) dokumentiert. Die so zur Darstellung kommende vermehrte Druckbelastung entspricht dem palpatorischen Befund.

Nach plantar prominente, sekundär entstandene, appositionelle Verknöcherungen, wie diese beispielsweise durch verbliebene Spongiosareste auftreten, waren nur vereinzelt Ursache für einen plantaren Belastungsschmerz.

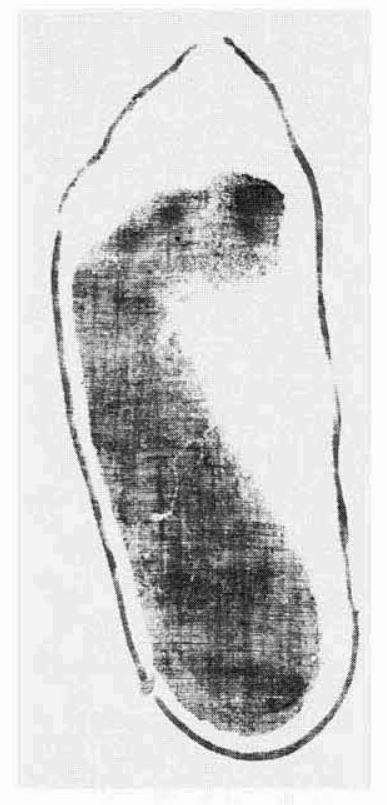

Abb. 4 Trittspur mit Darstellung der vermehrten Druckbelastung unter den nach plantar prominenten Metatarsalestümpfen nach Mittelfußköpfchenresektion (in der a.p. Röntgenaufnahme zeigte sich ein regelrechtes Alignement)
Ob die Zehenstellung postoperativ passager mit Kirschner-Draht oder mit einem Tennisschlägergips fixiert wurde, ergab keinen signifikanten Unterschied in Bezug auf das kosmetische Langzeitergebnis. Diejenigen Patienten, die nach der initial postoperativen Zehenfixation über mehr als 6 Wochen selbsttätig einen zehenschienenden Verband anlegten, wiesen in der Nachkontrolle eine bessere Zehenstellung auf als diejenigen, die nur kürzere Zeit eine Zehenschienung vornahmen.

Als bemerkenswert erwies sich die Bedeutung der Fußgymnastik für die Zehenbeweglichkeit. Von 20 Patienten, die regelmäßig eine Fußgymnastik durchführten, konnten lediglich zwei ihre Zehen nicht bewegen und kraftvoll gegen den Boden drücken, während der größte Teil $(90 \%)$ eine gute oder zumindest zufriedenstellende Zehenbeweglichkeit zeigte und die Zehen beim Abrollvorgang einsetzte. Von den Patienten, die keine Fußgymnastik durchführten, konnten $59 \%$ die Zehen überhaupt nicht bewegen.

\section{Diskussion}

Die Mittelköpfchenresektion nach Lelièvre zielt darauf, bei ausgeprägten Vorfußdeformitäten mit massiven Gelenkveränderungen die schmerzhaften Kontaktpunkte zu beseitigen und entsprechend dem Prinzip der Resektionsarthroplastik einen funktionellen Kontakt zwischen Zehen und Mittelfußstümpfen herzustellen (4). Das Belassen der Grundgliedbasen bei Digitus II-V bietet gegenüber der Methode von Clayton (2) den Vorteil, daß bei den oft gestörten Durchblutungsverhältnissen keine weiteren Traumatisierungen mit möglicher Beeinträchtigung der zirkulatorischen Situation entstehen.

Der plantare Zugang hat sich für das operative Vorgehen als günstiger erwiesen. Ein wesentlicher Vorteil liegt darin, daß die entzündlich veränderten Bursen unter den Metatarsaleköpfchen entfernt werden können. Außerdem gestaltet sich die Präparation beim plantaren Zugang wesentlich einfacher. Die nach plantar tiefer getretenen, prominenten Mittelfußköpfchen mit der nach plantar vorgewölbten Gelenkkapsel können bei dem deutlich atrophierten Fettsohlenpolster gut erreicht werden. Dagegen ist beim dorsalen Zugang die oft vorliegende Subluxation oder vollständige Luxation der Zehengrundgliedbasen nach dorsal über die Köpfchen zusätzlich hinderlich.

Ein weiterer Vorteil des plantaren Zuganges besteht darin, daß die Metatarsalestümpfe zur Belastungsseite hin angeschrägt und geglättet werden können, damit keine nach plantar hervorstehenden, schmerzverursachenden Knochenvorsprünge verbleiben.

Es ist unbedingt darauf zu achten, daß die Narbe nach distal, außerhalb der Belastungsfläche zu liegen kommt. Bei der von Tillmann (10) befürworteten Dermodese liegt die Narbe bei vollständiger Exision des Verschwielungsbereiches häufig in der Belastungsfläche. Außerdem besteht bei der Dermodese die Gefahr einer erhöhten Gewebespannung. Dadurch können postoperativ Wundheilungsstörungen auftreten. Nach unseren Beobachtungen können diese Probleme bei plantarem Zugang mit der Gocht'schen Schnittführung und Narbenlokalisa- 
tion distal der Metatarsalestümpfe vermieden werden. Wir teilen die Auffassung von Rhinelander (zit. nach Marmor, 7), daß die plantaren Schwielen postoperativ von selbst verschwinden, ohne daß eine Exision erforderlich ist. Gerade im Rückgang der Schwielen zeigt sich die Effektivität des operativen Verfahrens. In der Nachuntersuchung wurde dies deutlich.

Zur Frage des günstigsten Längenverhältnisses zwischen erstem und zweitem Metatarsale finden sich in der Literatur unterschiedliche Angaben: Nach Siegrist (8) darf das präoperative Längenverhältnis zwischen erstem und zweitem Strahl nicht geändert werden. Es muß postoperativ genauso fortbestehen. Lelièvre (6) wie auch Clayton (2) streben die gleiche Länge zwischen erstem und zweitem Metatarsale an. Vom zweiten bis fünften Strahl soll dann ein abfallendes Alignement geschaffen werden. Gschwend (4) beobachtet die günstigsten Ergebnisse bezüglich Schmerzfreiheit und guter Belastbarkeit des Vorfußes bei Plus-/Minus-Index und griechischer Zehenform. Eine Insuffizienz des ersten Strahles und eine Überlastung des zweiten Strahles fand er besonders bei Minus-Index und griechischer Zehenformel.

Die in der hiesigen Untersuchung vorgefundene höhere subjektive Zufriedenheit bei postoperativem Alignement mit längerbelassenem ersten Metatarsale ist am ehesten durch die erhaltene Belastungsfähigkeit des ersten Strahles zu erklären. Die deutlich tiefer getretenen mittleren Metatarsalia stehen aufgrund ihrer knöchernen Fixation in der Lisfranc'schen Gelenklinie auch mit ihren Stumpfenden relativ weit nach plantar vor (Abb. 4). Zeigt sich in der a.p. Röntgenprojektion eine gleiche Länge von erstem und zweitem Metatarsalestumpf, so steht dabei der Stumpf des zweiten Metatarsale weiter nach plantar in die Belastungsfläche und ist damit vermehrt den Auftrittsdruckkräften ausgesetzt. Beim Plus-Idex ist der zweite Strahl aufgrund der Verkürzung relativ entlastet und der Bodendruck vermehrt auf den stärkeren, besser belastbaren ersten Strahl verlagert. Von daher kann eine Nachresektion der Metatarsalestümpfe zwei bis vier auch bei unauffälligem Alignement in der a.p. Röntgenprojektion notwendig sein, um eine gleichmäßigere Druckbelastung unter den Metatarsalestümpfen zu erzielen. Von Lelièvre (6) und Bischofberger (1) wird die Nachresektion in diesen Fällen ausdrücklich als Korrektureingriff empfohlen. Die somit bewirkte Belastungsverlagerung auf die physiologischen Belastungspunkte unter erstem und fünftem Metarsale ist die Voraussetzung für eine regelrechte Schrittabwicklung (3).

Da bei den hier untersuchten Fällen nur vereinzelt sekundär entstandene, nach plantar prominente, appositionelle Verknöcherungen die Ursache für einen plantaren Belastungsschmerz waren, kann die Angabe von Tsakmaklis (11) nicht bestätigt werden, der eine direkte Beziehung zwischen appositioneller Verknöcherung und Gehbeschwerden sieht. Die hier vorgefundenen Verknöcherungen waren röntgenologisch unterschiedlich ausgeprägt und in fast allen Fällen ohne funktionelle Auswirkung.

Sekundären Zehenfehlstellungen muß durch operative und insbesondere postoperative Maßnahmen begegnet werden. Das Ziel der postoperativen Zehenfixation ist es, eine gute Stellungskorrektur der Zehen zu halten und durch Distraktion einen Kontakt der Gelenkpartner zu verhindern, um nach fibröser Verfestigung das Prinzip der Resektionsarthroplastik zu verwirklichen und eine sekundäre Zehenfehlstellung zu vermeiden. Die beiden hier angewandten Verfahren der passageren Fixation mit parossären Kirschnerdrähten sowie die Zügelung im Tennisschlägergips (Abb. 1) dienen lediglich als früh postoperative Fixationsmaßnahme.

Der sogenannte Tennisschlägergips bringt einige Nachteile für die Handhabung und den Behandlungsverlauf mit sich. Er muß am Ende der Operation mit erheblichem Aufwand angelegt werden. Die Zehen müssen mit Schlaufen, die an der Nagelplatte fixiert sind, aufgehangen werden. Oft ist es nur unzureichend möglich, die Zehen durch Zug in der Stellung auszurichten. Ein erheblicher Nachteil ist die Bedeckung der Wunde durch den Gips. Dadurch ist die Gefahr gegeben, daß bei den ohnehin gehäuften Wundheilungsstörungen durch eine so geschaffene abgeschlossene Kammer Wundheilungskomplikationen auftreten. Die Wundkontrolle ist durch den Gips mit Zehenfixation ebenfalls deutlich erschwert. Für die Mobilisierung ist der große Gips hinderlich.

Aufgrund dieser Umstände ist die initial postoperative Kirschnerdrahtfixation $\mathrm{zu}$ bevorzugen. Durch ihre parossäre Lage an den Zehen und im Weichteilgewebe des Fußes halten sie die Resektionsbezirke auf Distanz und schienen die Zehen. Da lediglich ein Schutzverband anliegt, ist die Wundkontrolle problemlos und Verbandswechsel können in üblicher Weise durchgeführt werden.

Zur Mobilisierung während der Kirschnerdrahtfixation hat sich die Fersensandale (Abb. 2) bewährt. Der Patient kann damit unter reiner Fersenbelastung auftreten bei Entlastung des Vorfußes, während die Sprunggelenke in Neutral-Null-Position eingestellt sind.

Die in der Nachkontrolle deutlich bessere Zehenstellung bei den Patienten, die nach der initial postoperativen Zehenfixation über mehr als sechs Wochen einen zehenschienenden Verband anlegten, ist am ehesten mit einer besseren bindegewebigen Verfestigung zu erklären. Die individuell gefertigte Zehenschiene nach Gschwend (5) stellt ein Optimum der Nachbehandlung dar (Abb. 3). Aufgrund der einfachen Handhabung durch Klettverschlüsse können die Schienen auch von Rheumatikern, die manuell eingeschränkt sind, leicht angelegt werden.

Da die Beweglichkeit der Zehen und damit die Gebrauchsfähigkeit maßgeblich von einer regelmäßig durchgeführten Fußgymnastik abhängt, sollte unbedingt zur Fußgymnastik angeleitet werden, um die Zehenfunktionsfähigkeit wieder herzustellen. Erst dann kann der Abrollvorgang in physiologischer Weise durchgeführt werden.

\section{Schlußbemerkung}

Bei dieser umfangreichen Vorfußoperation, die verschiedentlich auch als funktionelle Vorfußamputation bezeichnet wird, bleibt herauszustellen, daß die 
Behandlungserfolge nicht nur vom operativen Vorgehen, sondern in besonderen Maße von der Nachbehandlung abhängig sind. Um postoperativen Deformitäten zu begegnen, sollte zum einen eine ausgefeilte Operationstechnik angewandt werden, die nach präoperativer Planung der Belastbarkeit Rechnung trägt, zum anderen gebührt Zehenfixation und Fußgymnastik Aufmerksamkeit, um die postoperativ erreichte Zehenstellung und -funktion langfristig so gut wie möglich zu wahren.

Angesichts der vielen guten Resultate können wir bei Berücksichtigung der aufgezeigten Behandlungsmaßnahmen bei ausgeprägten Vorfußdeformierungen unbedingt zu dem Eingriff der Mittelfußköpfchenresektion nach Lelièvre raten, um einer Immobilität zu begegnen.

\section{Literatur}

${ }^{1}$ Bischofberger, R., N. Gschwend: Ergebnisse der Vorfußkorrektur nach Clayton. In: Der Vorfuß (hrsg. von P. Scholder). Bern, Huber 1970

${ }^{2}$ Clayton, M. L.: Surgery of the Lower Extremity in Rheumatoid Arthritis. J. Bone Joint Surg. 54 A (1963) 1517-1523

${ }^{3}$ Grifka, J.: Torsionswirkungen der Schuhsohlenrolle - Biomechanische Betrachtung. Z. Orthop. 124 (1986) 772-774

${ }^{4}$ Gschwend, N., M. Barbier, W. R. Dybowski: Die VorfußkorrekturHäufigkeit und Bedeutung der Zehen- und Metatarsalindices. Arch. orthop. Unfall-Chir. 88 (1977) 75-85

${ }^{5}$ Gschwend, N.: Persönliche Mitteilung

${ }^{6}$ Lelièvre, J.: Pathologie du pied. Masson, Paris 1971

7 Marmor, $L$.: Rheumatoid Deformity of the Foot. Arthr. and Rheum. 6 (1963) $749-755$

${ }^{8}$ Siegrist, H.: Unsere Erfahrungen mit dem Alignement nach Lelièvre. In: Der Vorfuß (hrsg. von P. Scholder). Bern, Huber 1970

9 Tillmann, K.: Rheumatische Fußveränderungen. Orthopädie 15 (1986) 344-348

to Tillmann, K.: Vorfußkorrektur. Orthopädie 2 (1973) 99-100

11 Tsakmaklis, P., W. Menke, A. Antoniadis: Langzeitergebnisse nach Metatarsalköpfchen- und Grundgliedbasen-Resektion in der Behandlung der Vorfußdeformität. OST (1986) 102-107

Dr. med. J. Grifka

Orthop. Universitätsklinik im St. Josef-Hospital

Gudrunstr. 56

D-4630 Bochum 